\title{
The future of mental health research in primary care settings
}

\author{
ANN-LOUISE KINMONTH AND CHRISTOPHER THOMPSON
}

\section{THE SCOPE OF RESEARCH}

Since the Declaration of Alma Ata (World Health Organization, 1978) there has been a steady move world wide for health care to be primary care led, with secondary care offering specialist knowledge and increasingly sophisticated investigation and treatment in support.

The vast majority of illness and disease is managed in primary care, but paradoxically, while general practice contributes the greatest activity and most prescriptions, it has until recently produced but a small fraction of the knowledge base for practice (Orme et al., 1990). Application of results from research in secondary care settings to primary care may be quite inappropriate due to differences in severity and natural history of disease among those patients reaching secondary care. At the end of the 20th Century we remain unclear about the epidemiology and response to treatment of many conditions in the community, and this applies particularly to mental health problems of all kinds.

There are two main reasons for this; on the one hand, is the complex nature of mental illness and disease, and the lack of aetiological clarity, leading to difficulty in defining cases at the research level in primary care. On the other, is the nature of primary care, it's organisation and delivery, which poses challenges to research design, intervention development and delivery, and valid and reliable outcome assessment.

As a result it remains an open question as to how to provide care most cost effectively to patients with schizophrenia through primary care. Equally the optimal detection and management strategies for the range of depression in primary care is unclear.

Indirizzo per la corrispondenza: Professor C. Thompson, Faculty of Medicine, Department of Psychiatry, University of Southampton, Royal South Hants Hospital, Brintons Terrace, Southampton SO14 0YG (UK).

Fax $+44-(0) 170-3234243$.
There are no studies of prevention of depression relapse from primary care, and the conceptual basis of somatisation and it's management in primary care is still at a very crude stage.

We will examine the obstacles to resolving questions such as these and map out the key areas for the future.

\section{The nature of mental health problems in primary care}

\section{a) Classifications}

Mental disorders usually present to primary care physicians with physical symptoms (Bridges \& Goldberg, 1985; Ustun \& Sartorius, 1995), and $40-50 \%$ of patients with psychiatric disorders have accompanying medical disorders (Goldberg \& Gates, 1996).

Diagnostic classifications such as DSM-IV, developed by the American Psychiatric Association, have proved useful in clinical practice, but may emphasise a dichotomy between physical and mental illness rather than interactions between them.

Both psychotic and non psychotic disorders are still classified largely at the syndrome level and with decreasing severity become increasingly hard to assess. As syndromes proliferate with each new revision, they appear to many GPs to have reduced utility for clinical practice and primary care research. So new schemes have to be produced for primary care. Like blood pressure or cholesterol, mental disorders such as depression are measured by rating scales (Thompson, 1989) and behave as continuous variables which must be dichotomised for clinical action (Rose, 1992).

The more extreme the presentation, the more confident we can be of defining a case, and the more able we are to carry out observational, analytic, or randomised controlled trials to establish cause or therapeutic effects. Less severe presentations are seen as the majority of cases in primary care and it is among these cases that it is hardest to achieve reliable evidence for practice. 


\section{b) Disease and illness}

Not only are the determinants of mental disease poorly understood, their manifestation as mental illness, experienced and presented by patients to practitioners, is affected by the individual and social context of the disease and the culture within which it is made manifest. Primary care practitioners are well used to managing presenting 'problems' rather than pathological diagnosis alone; and assessing the physiological, psychological and social factors contributing to the illness or predicament of the individual. (Stewart et al., 1995).

In research terms however, it is only with the increasingly sophisticated approach to measurement that is resulting from the emergence of health services research as a reputable discipline (Hiatt \& Goldman, 1994) that we are moving towards the ability to measure costs and benefits of different management strategies spanning the perspectives of the individual, the health service and society as a whole.

Thus for example, a modern trial of management of depression in primary care, will include not only validated scales for defining a case and it's outcome in somatic, psychological and cognitive terms (Treatment of Depression, 1993), but also a range of measures of cost and benefit in the broadest sense from financial costs to society, to impact on the drug side-effects, treatment satisfaction, quality of life and functional status.

It is only as we gain the results from these trials that we will begin to have confidence to make rational judgements about the use of scarce resources for mental health and it's opportunity costs.

The Nature of Primary Care as a setting for development and evaluation of mental health care strategies

\section{Organisation}

There are major international variations in the way that primary care is purchased and provided which affect the apparent patterns of mental health care. The major variations depend on whether the primary care system is distinct from the secondary care system, (with primary care physicians acting as gatekeepers' to secondary care) or not, and the extent to which primary care practitioners are paid on a capitation or fee for service basis (Starfield, 1992).
In national health services where capitation rates form a large part of primary care practitioners income, activity tends to be lower, and where item for service is paid, activity tends to be greater. In private primary care systems activity is related to ability and willingness to pay.

Anticipatory or preventative care can be associated with socialised systems with defined responsibilities for the health of a registered population, or with private systems on a personal paying basis. These variations are magnified by the characteristics of mental illness; those of an anxious disposition or sensitive to the slightest distress of body and mind may consult frequently (especially if money is no object) while those in need of systematic health care may only consult in crisis if at all (Westhead, 1985).

Research on the incidence, prevalence and natural history of mental disorder and it's management in the community, must take account of these variations. They must be considered in judging the appropriateness of a sampling frame made up of primary care consultations or of community lists, such as the electoral roll. They must also be considered when judging apparent variations in mental health between countries which may be as much as a reflection of population selection as of true population difference.

The recent WHO study of mental illness in general health care across the world illustrates the difficulties involved (Ustun \& Sartorius, 1995; Goldberg \& Gates, 1996). The researchers aimed to avoid poor research process by choosing 15 centres with established records of mental health research. All centres used the same methodology, including 3 measures of mental disorder presented in primary care consultations; assessment of psychological symptoms by the patient, by the practitioner and by a research interviewer. 25,916 patients filled in the General Health Questionnaire (Goldberg \& Williams, 1988) and 5438 (random sample) were interviewed using the primary care version of the Composite International Diagnostic Interview (Robins et al., 1988) and a disability scale.

This study has confirmed the wide variation in apparent prevalence of mental disorders amongst consecutive attenders diagnosed according to ICD-10 criteria. The mean prevalence was high at $24 \%$ (SE 0.6) but this hides a variation of from $53.5 \%$ (SE 3.0) in Santiago de Chile to $9.7 \%$ (SE 1.2) in Shanghai.

Choosing centres to achieve reliable research data limited their representativeness and there is uncertainty about the ability of the CIDI-PC to detect cul- 
turally specific ways of expressing distress. Moreover, it did not prove possible to devise a generalised measure of social depriviation across countries, and the varying threshold for consulting with physical and mental complaints across countries is not clear. These difficulties are compounded when interventions and their outcomes are added to descriptive epidemiology.

In general terms, studies that are strongest on the development of practical intervention strategies are weakest on research design and vice versa; the more concessions that are made to optimising an intervention, the more difficult it is to achieve a rigorous randomised design.

\section{Process of care}

Despite it's difficulties, the WHO study provided some important evidence of the effects of the process of care on mental health. Across the world primary care physicians agreed that $48.9 \%$ of the patients classified as cases by the research interview had a psychological disorder. However, where patients usually saw the same doctor for care, and had an appointment, and the doctor felt responsible for follow up or referral and kept records of the visit, agreement with research interview was higher than in other places $(54 \%$ v's $27 \%)$.

This emphasises the importance in research in primary care of the process as well as the structure of care. More patient centred care, where the practitioner aims to manage the patients concerns alongside the practitioner's concerns, has been shown to relate to the detection and assessment of psychological disorders (Goldberg \& Huxley, 1992) and to increased satisfaction, drug adherence and resolution of symptoms among patients (Stewart, 1995).

\section{Workload and it's distribution}

The consultation length in primary care varies from 5 minutes to 30 minutes and is currently $8 \mathrm{~min}$ utes in the UK, with the average patient being seen 3 times annually and $85 \%$ of patients seen in one year (OPCS, 1995). This workload is increasingly managed by not only primary care physicians, but practice nurses and in the case of mental illness, community psychiatric nurses and a range of counsellors.

The move from reactive care for patients' symptoms, to proactive care for prevention and systema- tic review of chronic disease, has created new workloads and roles within the team, and has demanded the development of detailed morbidity and age sex records in primary care as the prerequisite for surveillance (Tudor Hart, 1988). This process is greatly supported by the growing availability of information technology in primary care.

The patterns of consultation, work roles and computer support, both offer an important area for research on the optimal delivery of care for people with psychiatric problems and provide the research environment in which they must be answered.

Primary care practitioners, whether in private practice or in contract with the state, have never enjoyed a career structure which includes academic work as an option, nor the financial subsidies available to hospitals to offset the extra service costs associated with research and teaching. The training of doctors and generalist nurses in mental health care in the community is still weak and the cost effectiveness of time intensive training programmes at both undergraduate and post-graduate level still needs to be established in randomised controlled trials.

Much is now being done to develop such effective training programmes (Maguire et al., 1986) and, before and after, or quasi experimental studies, suggest that teaching of communication skills at the undergraduate level can lead to persistent changes in medical behaviour and teaching of skills in the detection and management of depression to GPs can reduce hospitalisation and drug costs and possibly reduce suicide rates (Rutz et al., 1989; 1992).

The biases inherent in those study designs, however, limit interpretation and randomised controlled trials now in process will provide more robust evidence (Thompson et al., in press).

The challenge for the future development of primary care as an effective research setting is thus threefold.

1. To integrate mental health into the mainstream of primary health care and to ensure that a cadre of academic practitioners, nurses and doctors is formed capable of articulating clear, soluble and relevant research questions from the primary care perspective and linking in with the research disciplines necessary to solve them; statisticians, health economists, psychologists, sociologists and anthropologists. The pressure on practitioners for service delivery throughout the world, and the variability in academic development makes this a major undertaking.

2. To develop the research infrastructure neces- 
sary for the conduct of robust pragmatic randomised controlled trials in primary care.

There are several examples of how this could be achieved (Thompson et al., in press), but no one model will be suitable for all settings nor for different types of study (psychological v's pharmacological for example). The multi-centred nature of RCTs in primary care requires the strong organisation of a central research team to control the study. This is inevitably expensive but essential. New publication requirements on RCTs imposed by JAMA and the BMJ include reporting of the number of patients considered for entry to randomisation (ie registered). For most RCTs in primary care, this information will be crucial to understand the representativeness of the sample. However, accurate registration of all eligible patients, including those not subsequently randomised, will require a consciousness of the research process in each participating doctor, which is rare in secondary care, let alone in primary care. An appropriate infrastructure will have to support GPs and other team members in such day-to-day aspects of trials, as well as getting the general strategy right.

3. To link research with teaching so that effective dissemination of good practice occurs.

There are several ways of achieving this, but they are insufficiently used. First the profession must feel some ownership of the information being disseminated, ie. it is learned better if coming from a member of their own profession rather than, for example, a health economist (Treatment of Depression, 1993). Audit programmes can be developed from research results, which encourages local application of evidence-based standards. Finally policy makers require research results and, over a limited but strategically important area, may be able to make speedy changes to practice.

In summary, research into clinical efficacy no less than health services and educational research must, in future, be carried out in general practice on representative samples of patients. This will require wide ranging changes in ethos, organisation, training and finance, to develop the necessary academic infrastructure in primary care settings. We believe that these changes will be soonest achieved by developing partnerships between generalist and specialist clinical and research providers, in specific areas such as mental health, as well as developing the academic infrastructure of primary care more generally.

\section{REFERENCES}

Bridges K. \& Goldberg D. (1985). Somatic presentations of DSMIII Psychiatric Disorders in Primary Care. Journal of Psychosomatic Research 29, 563-569.

Goldberg D. \& Gates R. (1996). Implications of the WHO study of mental illness in general health care for training primary care staff. British. Journal of General Practice 46, 483-485.

Goldberg D. \& Huxley P. (1992). Common Mental Disorders: a Biosocial Model. Routledge: London.

Goldberg D. \& Williams P. (1988). A Users Guide to the General Health Questionnaire. NFER-Nelson: Windsor.

Hiatt H. \& Goldman L. (1994). Making medicine more scientific. Nature 371, 100.

Maguire P., Fairbairn S. \& Fletch C. (1986). Consultation skills of young doctors. Benefits of feedback training as medical students persists. British Medical Journal 292, 1573-1578.

OPCS (1995). Morbidity Statistics from General Practice. Fourth national study 1991-1992.

Orme M., Sjoqvist F., Birche J. et al. (1990). The teaching and organisation of clinical pharmacology in European medical schools. European Journal of Clinical Pharmacology 38, 101105.

Robins L., Wing L., Wittchen H-U., Helzer J.E., Babor T.F., Burke J., FarmerA,, JablenskiA., Pickens R., Regier D.A. et al. (1988). The composite international diagnostic interview. $A r-$ chives of General Psychiatry 45, 1069-1077.

Rose G. (1992). The Strategy of Preventive Medicine. Oxford University Press: London.

Rutz W., Von Knorring L. \& Walinder J. (1992). Long term effects of an educational programme for GPs given by the Swedish Committee for the Prevention and Treatment of Depression. Acta Psychiatrica Scandinavica 85, 83-88.

Rutz W., Walinder J., Eberhard G., Holmberg G., von Knorring A.L., von Knorring L., Wistedt B. \& Aberg-Wistedt A. (1989). An educational programme on depressive disorders for GPs on Gotland: background and evaluation. Acta Psychiatrica Scandinavica 79, 19-26.

Starfield B. (1992). Primary Care. Oxford University Press: London..

Stewart M. (1995). Studies of health outcome and patient-centred communication. In Patient-Centred Medicine (ed. M. Stewart et al.), pp. 185-190.

Stewart M. et al. (1995). Patient-Centred Medicine. Sage Publications: London.

Treatment of Depression in Primary Care (1993). Effective Health Care Bulletin, March 1993, No 5, 1-12.

Thompson C. (1989). The Instruments of Psychiatric Research. Wiley John \& Sons: Chichester.

Thompson C., Stevens L., Ostler K. et al. (in press). The Hampshire Depression Project. A methodology for assessing the value of general practice education in depression. Journal of Methods in Psychiatric Research.

Tudor Hart J. (1988). A New Kind of Doctor. Merlin Press Limited:. London.

Ustün B. \& Sartorius N. (1995). Mental Illness in General Health Care. John Wiley: Chichester.

Westhead J.N. (1985). Frequent attenders in general practice: medical, psychological and social characteristics. Journal of Royal College of General Practitioners 35, 337-340.

World Health Organization (1978). Thirtieth Annual Report. Primary Health Care. WHO: Geneva. 